\title{
Measuring ataxia: quantification based on the standard neurological examination
}

\author{
N C Notermans, G W van Dijk, Y van der Graaf, J van Gijn, J H J Wokke
}

\begin{abstract}
To quantify ataxia in a simple way four tests were developed and analysed, based on the neurological examination: a tapping test for the arms (test 1), another one for the legs (test 2), a quantified finger-to-nose test (test 3 ), and a modified Romberg test (test 4). All tests were performed by 115 volunteers, 13 patients with cerebellar ataxia and 25 patients with sensory ataxia due to neuropathy. The test-retest repeatability was excellent. Tests 1,2 and 4 were age-dependent, with lower scores above age 65 . On test 1,2 and 4 both groups of patients performed worse than controls; the correlation with the ataxia scale of NobileOrazio and the modified disability Rankin scale was good $(P<0.05)$. Although test 3 could differentiate between sensory and cerebellar ataxia, it was not useful for quantifying the degree of ataxia. To determine the practical value of the four tests, 11 patients performed the tests for a second time after a follow up period of 16 months. The results indicate that tests 1,2 and 4 are sensitive for the detection of ataxia and of changes in its severity.
\end{abstract}

(F Neurol Neurosurg Psychiatry 1994;57:22-26)

The neurological examination usually lacks the accuracy to detect small changes in muscle strength, sensation or coordination needed to evaluate the clinical course of neurological diseases and the efficacy of treatment. Many tests have been developed to quantify motor and sensory function. ${ }^{12}$ Tests for measuring ataxia are rare, and often very complicated. ${ }^{3-7}$ Although the assessment of the severity of illness should ultimately rely on integrated measures of function such as activities of daily life or the degree of dependency, the measurement of separate impairments may be useful for preliminary studies. ${ }^{89}$

This study aimed to develop a set of tests suitable for quantifying ataxia in a simple way. It was not our purpose to design a diagnostic test for ataxia, as some tests may be influenced by other factors. The tests should be easy to use in an outpatient setting and should also be comparable with the neurological examination normally used for assessing coordination of movement (for example, finger-to-nose test, diadochokinesia and Romberg's test).

\section{Materials and methods}

Controls

A total of 115 volunteers (60 men, 55 women; mean age 43 years SD 19 years) participated. All volunteers were in good health, without any underlying disease or disability which would have affected test scores. In particular no neurological disorder, rheumatoid arthritis or visual impairment was present. Nobody used any medication or drugs. There was no alcohol abuse.

\section{Patients}

We examined 38 patients of whom 13 had cerebellar ataxia (12 men, 1 woman; mean age 43 years SD 9 years) and 25 a sensory polyneuropathy and ataxia (18 men, 7 women; mean age 64 years SD 7 years). In all patients ataxia was diagnosed on clinical examination. We diagnosed a patient as having ataxia if there was a disturbance of voluntary movements, not caused by muscle weakness and consisting of: hypermetry, intention tremor, dysdiachokinesia, position sense disturbance, broad-based gait or an abnormal Romberg test. ${ }^{10}$

The cause of the cerebellar ataxia was a bilateral hemangioblastoma in one patient, early onset cerebellar ataxia with retained tendon reflexes in three patients, autosomal dominant cerebellar atrophy in three patients and isolated late onset cerebellar ataxia in five patients. ${ }^{11}$ The patients with cerebellar ataxia had no muscle weakness.

Of the 25 patients with a sensory polyneuropathy, ten had a monoclonal gammopathy of undetermined significance..$^{12}$ In seven of these ten patients only mild peroneal weakness was found (MRC grade $4+$ ). No signs of cerebellar dysfunction were found in any of them. The other 15 patients with a sensory polyneuropathy were diagnosed as having a chronic idiopathic sensory polyneuropathy. ${ }^{13}$ The most prominent feature of the patients with a sensory neuropathy was a sensory ataxia. This sensory ataxia was shown by a broad-based gait, inability to stand on one leg or with the feet close together. None of these patients showed muscle weakness or cerebellar signs. The extensive clinical work up of a patient suspected of having a polyneuropathy is described elsewhere. ${ }^{2}$ As in the control group, none of the patients with cerebellar ataxia or polyneuropathy had complaints about musculoskeletal or joint dysfunction. In addition there were no signs of pyramidal or extrapyramidal dysfunction. 

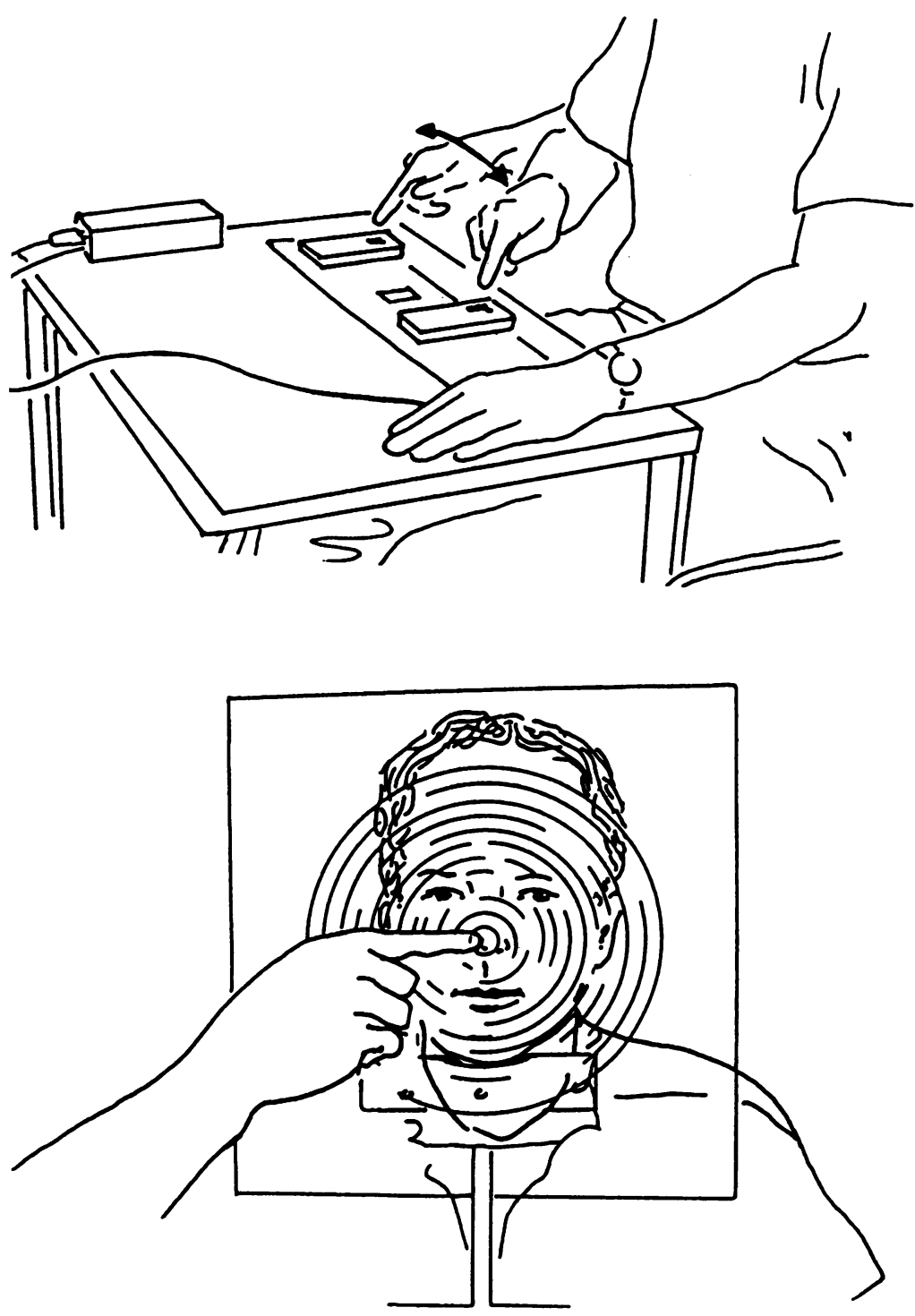

Figure $1 A$ The tapping test for the arms (top); $1 B$ The quantitative finger-to-nose test (bottom). ticipant is seated on a standard chair in front of the device which is flat on the floor. This test is performed in a similar fashion as test 1 , but with the feet instead of the hands.

Test 3

A quantitative finger-to-nose test. Participants are seated in front of a transparent screen. The screen is placed against the tip of the nose, with the nose centred in the middle of the screen. Subjects are asked to put their index finger to the tip of their nose, first with their eyes open, then with their eyes closed. The distance between the tip of the nose and the point where the index finger touches the screen is measured in centimetres, by means of concentric lines drawn on the screen (fig 1b).

\section{Test 4}

A quantitative Romberg test. Participants are asked to perform the Romberg test three times: (a) with their feet $15 \mathrm{~cm}$ apart (test 4A), (b) with their feet close together (test 4B) and (c) with the feet in line (heel to toe) (test 4C). The time is measured from the moment the participant closes the eyes until they open their eyes or are likely to fall. Every subject is stimulated to continue the test for as long as possible, but for practical reasons the Romberg tests were limited to a maximum of 60 seconds.

The tests 1-3 were performed four times; twice on the dominant and twice on the nondominant side. The results of each side are presented as the mean value of the two attempts.

\section{Follow up}

All four tests were performed a second time in 11 patients (seven with cerebellar ataxia, four with sensory ataxia) after a follow up period of 16 months. Practical reasons determined the selection for the follow up study. All patients were rated on the ataxia scale described by Nobile-Orazio et $a l^{14}$ and on the modified disability Rankin Scale (table 1). ${ }^{15}$ The latter was chosen rather than an activity of daily living scale, as the ataxia does not interfere with self-care in all patients.

Table 1 Clinical severity of disease, according to the modified Rankin scale and the ataxia scale according to Nobile-Orazio

\section{Test 1}

A tapping test for the upper limbs. The test uses a device consisting of two push buttons placed at a fixed distance of $35 \mathrm{~cm}$ apart, connected to a counter. The participant is placed behind a standard desk in front of the device and is asked to push the left and right buttons alternately with the index finger, as fast as possible. The counting starts as soon as the first button is pushed, and stops automatically after 15 seconds (fig $1 \mathrm{a}$ ).

Test 2

A tapping test for the lower limbs. The test uses a device consisting of two push pedals placed at a fixed distance of 35 centimetres apart, again connected to a counter. The par-
Modified Rankin scale (disability scale)

1 Asymptomatic

2 Minor disability; symptoms that lead to some restriction of life style, but do not interfere with the patients' capacity to look after themselves

3 Moderate disability; symptoms that significantly interfere with life style or prevent totally independent existence

4 Moderately severe disability; symptoms that clearly prevent independent existence, although patient does not need independent existe

5 Severely disabled; totally dependent requiring constant attention

Nobile-Orazio (ataxia scale)

0 Normal-stand on one foot with eyes closed

1 Stand/walk normally with eyes closed normally with eyes open

3 Stand/walk with some swaying with eyes open

$4 \mathrm{Stand} / \mathrm{walk}$ on a large base with eyes open

5 Standing/walking impossible without support
2 Stand/walk with minor swaying with eyes closed, but 


\section{Statistical analysis}

The results of test scores can be considered as an ordinal scale. The results are therefore presented in percentiles. We used the following non-parametric tests: Spearman's rank correlation coefficient, Wilcoxon signed rank test and Mann-Whitney test. Only $\mathrm{p}$ values below 0.05 were considered significant. Testretest repeatability was measured by calculating the coefficient of repeatability. ${ }^{16}$ This coefficient is defined as twice the standard deviation of the differences between two measurements. All test scores of the patients were compared with an age-matched group of controls.

\section{Results}

Test-retest repeatability

The possible influence of intra-individual biological variabilities, motivation and learning effects was examined in nine healthy volunteers. All four tests were executed at the same time of day on three consecutive days and after instruction by the same examiner (GWvD). The test results on these three occasions were the same (Wilcoxon signed rank test, $\mathrm{p}<0.0001$ ).

To assess the overall reproducibility of the tests, all controls and patients performed

Table 2 Test-retest repeatability

\begin{tabular}{|c|c|c|c|c|c|c|}
\hline \multirow[b]{2}{*}{ Test } & \multicolumn{2}{|c|}{ Controls } & \multicolumn{2}{|c|}{ Cerebellar ataxia } & \multicolumn{2}{|c|}{ Sensory ataxia } \\
\hline & $M D$ & $C R$ & $M D$ & $C R$ & $M D$ & $C R$ \\
\hline $\begin{array}{l}1 \mathrm{D} \\
\text { 2D } \\
3 \mathrm{DO} \\
3 \mathrm{DC} \\
4 \mathrm{~A} \\
4 \mathrm{~B} \\
4 \mathrm{C}\end{array}$ & $\begin{array}{l}0.9 \\
0 \cdot 8 \\
0 \cdot 1 \\
0 \cdot 1 \\
0 \\
0 \\
8 \cdot 3\end{array}$ & $\begin{array}{c}6 \cdot 8 \\
6.5 \\
1 \cdot 4 \\
1 \cdot 7 \\
0 \\
0 \\
13 \cdot 7\end{array}$ & $\begin{array}{l}1 \cdot 0 \\
1.0 \\
0.2 \\
0.3 \\
2.3 \\
2 \cdot 3 \\
1 \cdot 8\end{array}$ & $\begin{array}{r}3.3 \\
6.5 \\
2.3 \\
2.3 \\
11 \cdot 2 \\
16.1 \\
8.0\end{array}$ & $\begin{array}{l}0.7 \\
0.5 \\
0 \\
0 \cdot 1 \\
1 \cdot 3 \\
0 \cdot 2 \\
0.2\end{array}$ & $\begin{array}{l}5 \cdot 6 \\
5 \cdot 6 \\
2 \cdot 1 \\
3 \cdot 0 \\
8 \cdot 3 \\
5 \cdot 4 \\
2 \cdot 6\end{array}$ \\
\hline
\end{tabular}

The test-retest repeatability in controls, cerebellar ataxia and sensory ataxia patients. $M D=$ mean difference; $\mathrm{CR}=$ coefficient of repeatability. $\mathrm{D}=$ dominant side; $\mathrm{O}=$ eyes open; $\mathrm{C}=$ eyes closed.

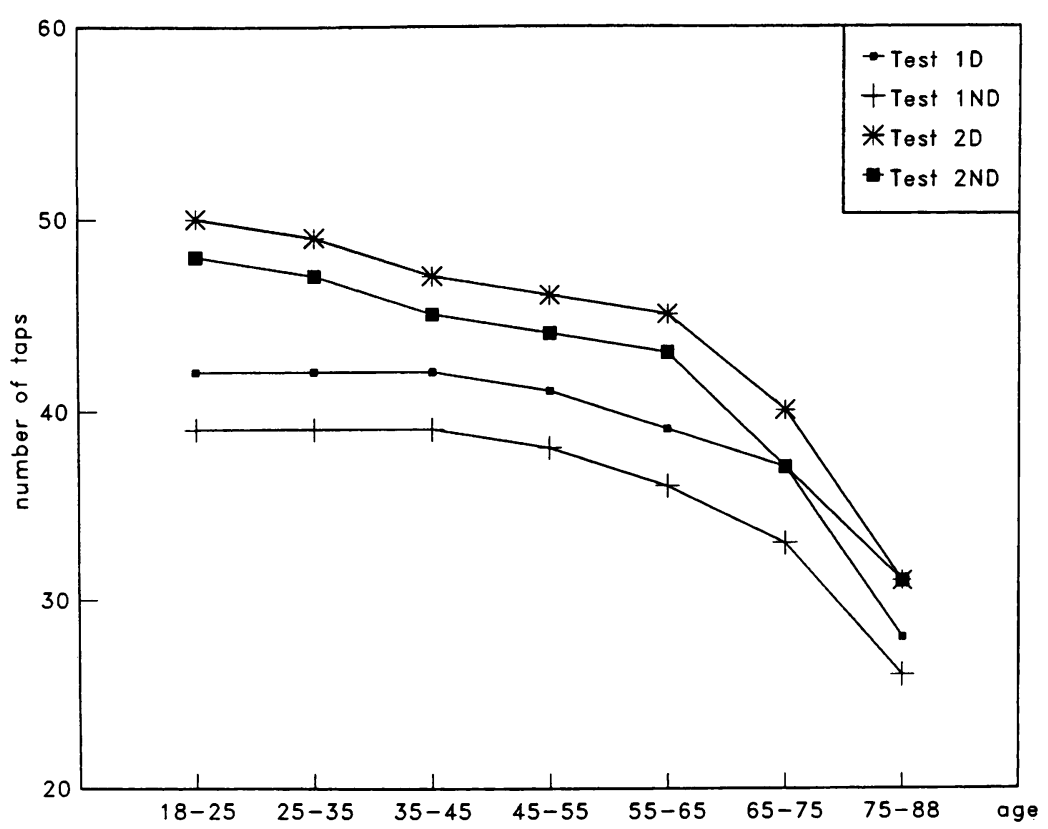

Figure 2 Relation between the 50-percentile value of the tapping tests' scores and age. every test twice. The agreement was summarised by calculating the mean differences between the two measurements and the standard deviation of these differences (table 2). This was done separately for the dominant and nondominant side. A good reproducibility was found for both tapping tests (1 and 2) and for the Romberg test (4A and 4B) (table $2)$. Because the test/retest reliability was the same for the dominant and the nondominant side, only the results of the dominant side are presented in table 2 .

Normal values in controls

None of the four tests showed significant gender related differences in the control group (Mann-Whitney test, $\mathrm{p}>0 \cdot 30$ ).

The tapping test scores were related to age (fig 2). The scores of tests 1 and 2 decreased significantly after 65 years of age (MannWhitney test, $p<0.001$ ). For the finger-tonose test (test 3 ) no influence of age was detected (Mann-Whitney test, $p=0 \cdot 4$ ). With regard to the Romberg test with both feet 15 $\mathrm{cm}$ apart (test $4 \mathrm{~A}$ ), all controls performed for the maximum time of 60 seconds. The Romberg test with feet close together (test 4B) could be performed for 60 seconds by all controls except two of the four volunteers who were older than 80 years. The test performance for the Romberg test with feet in line (test 4C), decreased with age: 18-40 years: median performance time $53 \mathrm{~s}$ (q1 $=$ $18, \mathrm{q} 2=60)$; $40-60$ years: median performance time $25 \mathrm{~s}(\mathrm{q} 1=14, \mathrm{q} 2=60) ; 60-80$ years; median performance time $10 \mathrm{~s}(\mathrm{q} 1=2$, q2 $=27$ ) (Spearman's rank correlation coefficient: $-0.34, \mathrm{p}<0.001)$.

Scores on the dominant side were significantly better than those on the nondominant side for the tapping tests ( 1 and 2; Wilcoxon, $p<0.001$, table 3 ). The quantitative fingerto-nose test (test 3) did not discriminate between the dominant and the nondominant side. Test performances of right-handed subjects (101 persons) were not different from those of left-handed subjects (14 persons).

Cerebellar and sensory ataxia patients

All four tests discriminated between the group of controls and the group of patients with cerebellar ataxia (Mann-Whitney test, $p<0.001$; table 3 ). Except for the finger-tonose test with eyes open, all tests also discriminated between controls and sensory ataxia patients (Mann-Whitney test, $\mathrm{p}<0.001$; table 3). The cerebellar ataxia patients performed significantly worse than the sensory neuropathy patients on the two tapping tests $(p<0.001)$ and on the fingerto-nose test with eyes open $(p<0.05)$. Cerebellar ataxia patients showed no significant difference between eyes open and eyes closed on the finger-to-nose test (Wilcoxon test, $p=0 \cdot 5)$. In contrast, the sensory neuropathy patients did significantly better on the finger-to-nose test with eyes open than with eyes closed (Wilcoxon test, $\mathrm{p}<0.005$ ). With regard to the Romberg test, the results confirmed the expected difference in diffi- 
Table 3 Test performances

\begin{tabular}{|c|c|c|c|c|c|c|}
\hline \multirow[b]{2}{*}{ Test } & \multicolumn{2}{|l|}{ Controls } & \multicolumn{2}{|c|}{ Cerebellar ataxia } & \multicolumn{2}{|c|}{ Sensory ataxia } \\
\hline & $50-\%$ tile & $10-90 \%$ tile & $50-\%$ tile & $10-90 \%$ tile & $50-\%$ tile & $10-90 \%$ tile \\
\hline $\begin{array}{l}\text { 1D } \\
\text { 1ND } \\
\text { 2D } \\
\text { 2ND } \\
\text { 3DO } \\
\text { 3NDO } \\
\text { 3DC } \\
\text { 3NDC } \\
4 A \\
4 \mathrm{~B} \\
4 \mathrm{C}\end{array}$ & $\begin{array}{l}41 \\
38 \\
48 \\
46 \\
1 \cdot 0 \\
1 \cdot 3 \\
1 \cdot 5 \\
1 \cdot 5 \\
60 \\
60 \\
32\end{array}$ & $\begin{array}{c}32-49 \\
32-45 \\
36-56 \\
35-54 \\
0 \cdot 5-2 \cdot 0 \\
0 \cdot 5-2 \cdot 0 \\
0 \cdot 5-3 \cdot 0 \\
0 \cdot 5-3 \cdot 0 \\
60-60 \\
60-60 \\
5-60\end{array}$ & $\begin{array}{c}21 \\
20 \\
25 \\
24 \\
2 \cdot 2 \\
2 \cdot 0 \\
2 \cdot 0 \\
2 \cdot 4 \\
60 \\
60 \\
0\end{array}$ & $\begin{array}{c}12-31 \\
11-31 \\
18-36 \\
15-34 \\
1 \cdot 0-4 \cdot 0 \\
1 \cdot 0-5 \cdot 5 \\
0 \cdot 5-6 \cdot 0 \\
1 \cdot 5-6 \cdot 0 \\
15-60 \\
0-60 \\
0-10\end{array}$ & $\begin{array}{c}30 \\
28 \\
34 \\
33 \\
1 \cdot 0 \\
1 \cdot 5 \\
2 \cdot 0 \\
2 \cdot 5 \\
30 \\
10 \\
0\end{array}$ & $\begin{array}{c}22-36 \\
20-35 \\
25-44 \\
25-39 \\
0-1 \cdot 5 \\
0-2 \cdot 5 \\
0 \cdot 5-4 \cdot 5 \\
1-4 \cdot 5 \\
0-60 \\
0-60 \\
0-5\end{array}$ \\
\hline
\end{tabular}

The 50-percentile of the scores for all four tests are presented for controls, patients with cerebellar ataxia and patients with sensory ataxia. The 10 to 90 -percentile range is also given (D: dominant side; ND: nondominant side; O: eyes open; C: eyes closed).

Table 4 Correlation coefficients of all three scoring systems

\begin{tabular}{|c|c|c|c|c|}
\hline \multirow[b]{3}{*}{ Test } & \multicolumn{2}{|c|}{ Cerebellar ataxia } & \multicolumn{2}{|c|}{ Sensory ataxia } \\
\hline & Rankin scale & $\begin{array}{l}\text { Nobile-Orazio } \\
\text { scale }\end{array}$ & Rankin scale & $\begin{array}{l}\text { Nobile-Orazio } \\
\text { scale }\end{array}$ \\
\hline & $\rho$ & $\rho$ & $\rho$ & $\rho$ \\
\hline $\begin{array}{l}\text { 1D } \\
\text { 2D } \\
3 \mathrm{DO} \\
3 \mathrm{DC} \\
4 \mathrm{~A} \\
4 \mathrm{~B} \\
4 \mathrm{C}\end{array}$ & $\begin{array}{l}0.9 \\
0.6 \\
0.4 \\
0.2 \\
0.7 \\
0.6 \\
0.8\end{array}$ & $\begin{array}{l}0.8 \\
0.6 \\
0.6 \\
0.4 \\
0.7 \\
0.7 \\
0.7\end{array}$ & $\begin{array}{l}0.8 \\
0.5 \\
0.5 \\
0.6 \\
0.7 \\
0.7 \\
0.2\end{array}$ & $\begin{array}{l}0.9 \\
0.6 \\
0.5 \\
0.6 \\
0.8 \\
0.7 \\
\underline{0.2}\end{array}$ \\
\hline
\end{tabular}

The Spearman's rank correlation coefficient $(\rho)$ between the three scoring systems: the present four tests, the modified Rankin scale and the ataxia scale of Nobile-Orazio, in patients with cerebellar ataxia and patients with sensory ataxia. The $p$ values of the underlined correlation coefficients are not significant $(p>0.05)$. All other $p$ values $<0.05$. D: dominant side; $O$ : eyes open; C: eyes closed.

culty between the three sub-tests $(4 A, 4 B, 4 C)$. The patients with sensory neuropathy tended to perform the Romberg test worse than the patients with cerebellar ataxia. Test $4 \mathrm{C}$ could not be performed by either category of patients with ataxia.

When the results were analysed, not at the level of the group but at that of the individual, three patients with sensory neuropathy showed scores on the two tapping tests that were within the range of those of 90-percentile of controls, whereas there was no overlap between controls and patients with cerebellar ataxia.

Comparison of scales

All patients were also graded on the ataxia scale of Nobile-Orazio and on the modified disability Rankin scale. These scores were then compared with the results of our own tests, by means of the Spearman's rank corre- lation coefficients. The correlation of the tests 1,2 and 4A-B with the ataxia scale of NobileOrazio and with the disability Rankin scale was good $(p<0.05 ;$ table 4$)$. Correlation coefficients of test 3 with the Nobile-Orazio ataxia scale and with the Rankin's disability scale were significant only for patients with a sensory ataxia $(p<0.01$; table 4$)$. Correlation coefficients of test $4 \mathrm{C}$ with the Nobile-Orazio ataxia scale and with the Rankin's disability scale were significant only for patients with a cerebellar ataxia $(p<0.01$; table 4$)$.

\section{Clinical application of the four tests}

Seven patients with cerebellar ataxia and four patients with a sensory neuropathy associated with a monoclonal gammopathy performed the four tests for a second time after a follow up period of 16 months; they were simultaneously graded on the modified Rankin scale and the ataxia scale of Nobile-Orazio (table 5). Five patients with cerebellar ataxia and three patients with sensory neuropathy reported a subjective deterioration of their writing abilities or of gait and stability in this period. The standard neurological examination or the Rankin scale did not reflect this deterioration in six of these eight patients. Our tests, however, confirmed the subjective impression in all eight patients. In the remaining two patients the deterioration was so severe that it could be detected on neurological examination; it also resulted in a lower grade on the Rankin scale (table 5). During the follow up period there was no change in the Nobile-Orazio scores for any of the patients.

\section{Discussion}

Quantifying the clinical phenomenon of ataxia is difficult. Only a few scoring tests for ataxia are available. ${ }^{3-7}$ Most of these tests, however, are difficult to perform, which makes them impractical for everyday clinical practice. Weakness may negatively influence a patient's performance when ataxia is measured. In addition, bradykinesia or pyramidal weakness will affect both tapping tests, while tremor and vestibular problems will disrupt test 3 and 4, respectively. The test results can also be impaired by other physical handicaps (for example, arthrosis or visual impairment), lack of motivation or effort, and by fatigue.

Table 5 Follow up study

\begin{tabular}{|c|c|c|c|c|c|c|c|c|c|c|}
\hline & \multirow[b]{2}{*}{ Patient } & \multicolumn{2}{|c|}{ Test $1 D$} & \multicolumn{2}{|c|}{ Test $2 D$} & \multicolumn{2}{|c|}{ Test $4 B$} & \multicolumn{2}{|c|}{ Rankin } & \multirow[b]{2}{*}{ Follow up } \\
\hline & & To & $T e$ & To & $T e$ & To & $T e$ & To & $T e$ & \\
\hline $\begin{array}{l}\text { Cerebellar } \\
\text { ataxia }\end{array}$ & $\begin{array}{l}1 \\
2 \\
3 \\
4 \\
5 \\
6 \\
7 \\
1 \\
2 \\
3 \\
4\end{array}$ & $\begin{array}{l}31 \\
25 \\
18 \\
22 \\
21 \\
26 \\
18 \\
34 \\
30 \\
27 \\
30\end{array}$ & $\begin{array}{l}27 \\
22 \\
16 \\
16 \\
21 \\
24 \\
20 \\
30 \\
22 \\
24 \\
30\end{array}$ & $\begin{array}{l}34 \\
39 \\
23 \\
31 \\
31 \\
21 \\
24 \\
38 \\
36 \\
36 \\
35\end{array}$ & $\begin{array}{l}32 \\
34 \\
18 \\
27 \\
26 \\
23 \\
20 \\
34 \\
26 \\
32 \\
35\end{array}$ & $\begin{array}{l}60 \\
60 \\
60 \\
60 \\
20 \\
60 \\
0 \\
60 \\
30 \\
0 \\
60\end{array}$ & $\begin{array}{l}60 \\
60 \\
30 \\
0 \\
10 \\
60 \\
0 \\
30 \\
0 \\
0 \\
60\end{array}$ & $\begin{array}{l}2 \\
3 \\
3 \\
3 \\
3 \\
3 \\
3 \\
2 \\
2 \\
2 \\
2\end{array}$ & $\begin{array}{l}2 \\
3 \\
4 \\
3 \\
3 \\
3 \\
3 \\
2 \\
3 \\
2 \\
2\end{array}$ & $\begin{array}{l}\text { deterioration } \\
\text { deterioration } \\
\text { deterioration } \\
\text { deterioration } \\
\text { deterioration } \\
\text { stable } \\
\text { stable } \\
\text { deterioration } \\
\text { deterioration } \\
\text { deterioration } \\
\text { stable }\end{array}$ \\
\hline
\end{tabular}

Mean values represent two attempts on each test, performed for the first time (To) and after a follow up of 16 months (Te) for the second time. In addition, the scores on the modified Rankin scale at To and Te are given. The follow up indicates the patients' subjective assessment of his/her clinical condition. D: dominant side. 
With our selection criteria some of these factors have been excluded.

We examined four aspects of ataxia. Both tapping tests are based on the usual tests for dysdiadochokinesia (test 1 for the arms, and test 2 for the legs). Our finger-to-nose test (test 3) was designed for quantifying an accurate, intentional movement. All three tests are concerned with nonequilibratory coordination. ${ }^{10}$ Our modified Romberg test (test 4) concentrates on equilibratory coordination. ${ }^{10}$

Learning effects do not appear to influence the results of our four tests as the overall intra-individual reproducibility was excellent. The influence of age and cerebral dominance on the results of both tapping tests may be explained because these tapping tests depend largely on speed and motor skills. It is understandable that the test results are worse for patients over 65 and for the nondominant side. In performing the tapping tests, controls did better than patients, and patients with sensory ataxia did better than patients with cerebellar ataxia. These findings accentuate the problem cerebellar ataxia patients have in performing motor skills and speed-related movements.

On the finger-to-nose tests with eyes open, the group of patients with cerebellar ataxia performed worse than patients with a sensory ataxia, but with the eyes closed, the two groups showed similar test scores. This confirms that patients with a sensory neuropathy gain more from visual guidance than patients with a cerebellar ataxia. Surprisingly, the scores with this test were similar for controls and for patients with a sensory ataxia (table 3). Furthermore, there was no correlation between the degree of disability and the deviation on the finger-to-nose test (table 4). Measuring the distance between the nose and the final placement of the index-finger on the transparent screen is probably not the best way of quantifying dysmetria. In the neurological examination, cerebellar dysmetria in this test is inferred from the typical inability to follow a straight line, whereas the final distance between fingertip and nose is less relevant.

Both ageing of the peripheral nervous system and sensory neuropathies may lead to loss or impairment of propriocepsis. This phenomenon may explain the decrease in performance of the Romberg tests in healthy elderly volunteers and in patients with a sensory neuropathy. ${ }^{17-19}$ The modified Romberg test (test 4C) proved of no additional value as even healthy volunteers were hardly able to perform this test for as long as 60 seconds.

Finally, the results of re-testing after 16 months in patients with cerebellar ataxia and sensory neuropathy indicate that tests 1,2 and 4 were very sensitive in the assessment of clinical deterioration. Using these tests, we were able to detect mild changes that were meaningful for the individual patient in activities of daily life. The Nobile-Orazio scale was not useful for monitoring the clinical deterioration in patients with cerebellar ataxia. On the other hand, this test was developed for quantifying sensory ataxia in patients with a sensory neuropathy associated with monoclonal gammopathy and not for cerebellar ataxia. ${ }^{13}$ In the patients with severe clinical deterioration, this was detected not only on neurological examination, but it was also reflected in worsening on the Rankin scale.

In conclusion, we have developed four tests to quantify the clinical phenomenon of ataxia. Particularly the two tapping tests and the Romberg test with the feet together reliably indicate the presence and severity of ataxia, and are useful for detecting mild changes in the course of the illness.

This study was supported by a grant from the Prinses Beatrix Fonds. We would like to thank Prof dr FGI Jennekens and Dr WHJP Linssen for critical review of the manuscript.

1 Munsat TL. Quantification of neurologic deficit. London: Butterworth, 1989 .

2 Notermans NC, Wokke JHJ, Jennekens FGI. Clinical work-up of the patient with a polyneuropathy. In: De Jong JMBV, ed Handbook of neurology. Amsterdam: Elsevier, 1991;253-70.

3 Poercher E, Barbeau A. Field testing of an ataxia scoring and staging system. $\mathcal{F}$ Can Sci Neurol 1980;7:339-45.

4 Graybiel A, Fregly AR. A new quantitative ataxia test battery. Acta Otolaryngol 1966;61:292-310.

5 Shimoama I, Nichoji T, Uemura $K$. The finger tapping test, a quantitative analysis. Arch Neurol 1990;47: test, a

6 Verkerk PH, Schouten JP, Oosterhuis HJGH. Measurement of the hand-coordination. Clin Neurol Neurosurg 1990;92:105-10.

7 Fillyaw MJ, Badger GJ, Bradley WG, et al. Quantitative measures of neurological function in chronic neuromuscular diseases and ataxia. $\mathcal{F}$ Neurol Sci 1989;92: 17-36.

8 Stein REK, Gortmaker SL, Perrin EC, et al. Severity of illness: concepts and measurements. Lancet 1987;ii: 1506-9.

9 Van Gijn J. Measurement of outcome in stroke prevention trials. Cerebrovasc Dis 1992;2(Suppl. 1):23-34.

10 De Jong RN. Coordination. In: De Jong RN, ed. The neurologic examination. London: Harper and Row, 1979: neurologic

11 Harding EA. Classification of the hereditary ataxias and paraplegias. Lancet 1983;i:1151-4.

12 Gosselin S, Kyle RA, Dyck PJ. Neuropathy associated with monoclonal gammopathies of undetermined significance. Ann Neurol 1991;30:54-61.

13 Notermans NC, Wokke JHJ, Franssen $\mathrm{H}$, et al Third meeting of the ENS, Lausanne, Switzerland. $f$ Neurol 1992;suppl(239) abstract, S89.

14 Nobile-Orazio E, Baldini L, Barbieri S. Treatment of patients with neuropathy and anti-MAG IgM Mproteins. Ann Neurol 1988;24:93-7.

15 Van Swieten JC, Koudstaal PK, Visser MC, Schouten HJA, Van Gijn J. Interobserver agreement for the assessment of handicap in stroke patients. Stroke 1988 ; 19:604-7.

16 Bland JM, Altman DG. Statistical methods for assessing agreement between two methods of clinical measureagreement between two metho
ment. Lancet 1986;8:307-10.

17 Goldberg JM, Lindblom W. Standardized method of determining vibratory perception threshold for diagnosis determining vibratory perception threshold for diagnosis
and screening in neurological investigations. $₹$ Neurol and screening in neurological investig

18 Jacobs JM, Love S. Qualitative and quantitative morphology of human sural nerve at different ages. Brain 1985; 108:897-924.

19 Albert ML. Clinical neurology of ageing. Oxford: Oxford University Press, 1992 